\title{
BEAT FREQUENCY AND VELOCITY VARIATION OF IONS IN A MAGNETIZED PLASMA SHEATH FOR DIFFERENT OBLIQUENESS OF THE MAGNETIC FIELD
}

\author{
B. R. Adhikari", S. Basnet, H. P. Lamichhane, R. Khanal \\ Central Department of Physics, Tribhuvan University, Kirtipur, Kathmandu, Nepal \\ *Corresponding author: b.r.adhikari@hotmail.com
}

(Received: August 8, 2018; Revised: December 22, 2018; Accepted: December 23, 2018)

\begin{abstract}
Beat frequency and velocity variation of ions in a magnetized plasma sheath has been numerically investigated by using a kinetic trajectory simulation (KTS) model for varying obliqueness of the external magnetic field in presence of an electric field. Angular dependence of mean value, maximum amplitude, damping constant, frequency of oscillation and beat frequency have been studied. As the obliqueness of the field changes the mean values, beat frequency as well as the maximum amplitude of the velocity components also change but frequency of oscillation remains almost the same.
\end{abstract}

Keywords: Plasma, Magnetized sheath, Bohm-Chodura condition, Kinetic theory, Beat frequency

\section{INTRODUCTION}

The study of beat frequency and velocity variation of ions in magnetized plasma sheath for varying obliqueness is one of the important problems for nearly all applications of plasma, where it is confined to a finite volume using magnetic fields (Chodura 1982, 1986, Riemann 1991, 2000). It is one of the most important problems of plasma. The correlative works, both of experiment and theory have been developed widely during the past several years and not yet fully understood (Chodura 1982, 1986, Riemann 1991, 2000, Hatami et al. 2008, Zou et al. 2009, Chalise \& Khanal 2012, Khoramabadi et al. 2011, Gyergyek \& Kovacic 2015). The sheath formed between magnetic plasma and a particle absorbing wall have received considerable amount of attention (Hatami et al. 2008, Zou et al. 2009, Chalise \& Khanal 2012, Khoramabadi et al. 2011, Gyergyek \& Kovacic 2015, Huang et al. 2015, Moulik \& Goswami 2015, Chalise \& Khanal 2015, Liberman et al. 2016, Chauhan et al. 2016). When the plasma is confined in any closed surface, the plasma interacts with the material surface so that the proper understanding of this interaction is very important in all plasma applications like in plasma confinement for fusion, sputtering, etching, surface treatment, etc (Chalise $\&$ Khanal 2012). Once the plasma-wall interaction is well understood it will be possible to control heat loading, energy transfer and particle flow towards the wall and overall bulk plasma behavior (Riemann 2000, Chalise \& Khanal 2012). The wall is generally charged up negatively due to the higher velocity of the plasma electrons as compared with that of the ions. Due to this reason, negative potential is developed in the absorbing wall which attracts the ions and repels part of the electrons, forming a positive space charge region in front of the wall, which is 'sheath'. For the formation and stability of such a sheath, the in-streaming ions at the sheath edge has to satisfy a condition called the Bohm criterion (Bohm
1949), which for a magnetized plasma is modified by Chodura (1982, 1986).

In presence of an oblique magnetic field, the charged particles entering into the plasma sheath region oscillate and it has been observed that the ion velocity shows beatlike nature. The beat nature shown by ion velocity in a magnetized plasma sheath has been reported and analyzed for different obliqueness of the magnetic field using a kinetic trajectory simulation (KTS) model (Khanal 2003) The importance of this study is to observe the changes in the particle dynamics as well as the particle wall interaction in magnetized plasma sheaths.

\section{METHODS AND MODEL}

KTS is an iterative method for numerically calculating self-consistent, time independent kinetic plasma states in some given bounded spatial region originally developed for non-magnetic cases (Khanal 2003) which was extended to include oblique magnetic field by Chalise and Khanal (2012). The characteristics feature of the KTS method is that the distribution functions of the ions are calculated directly, by solving the related kinetic equations along the respective collisionless particle trajectories. The electron density profile is still obtained analytically considering its cut-off Maxwellian distribution by the negative wall.

The fundamental equation which $f(\vec{r}, \vec{v}, t)$ has to satisfy is the Boltzmann equation (Chalise \& Khanal 2012).

$$
\frac{\partial f}{\partial t}+\vec{v} \cdot \nabla f+\frac{\vec{F}}{m} \cdot \nabla_{v} f=\left(\frac{d f}{d t_{c}}\right)
$$

Where, $\vec{F}$ is the force acting on the particles and $\left(\frac{d f}{d t}\right)_{c}$ is the time rate of change of $f$ due to collisions. The symbol $\nabla$ stands for the gradient in $(x, y, z)$ space and $\nabla_{v}$ 
stands for the gradient in velocity space, and $f(\vec{r}, \vec{v}, t)$ is a velocity distribution function. In collisionless cases the equation is called the Vlasov equation:

$$
\frac{\partial f}{\partial t}+\vec{v} \cdot \nabla f+\frac{q}{m}(\vec{E}+\vec{v} \times \vec{B}) \cdot \nabla_{v} f=0
$$

In the KTS method we solve the kinetic equation along with other basic equations describing the plasma for given boundary and initial conditions. The distribution function at any point along the trajectory can be obtained if its value at one point (i.e., at the boundary) is known. Then density of the species ' $s$ ' is given by

$$
n^{s}(\vec{x})=\int_{-\infty}^{\infty} d^{3} v f^{s}(\vec{x}, \vec{v})
$$

The space charge density is defined as

$$
\rho(\vec{x})=\sum_{s} q^{s} n^{s}(\vec{x})
$$

The electrostatic potential $\varphi(\mathrm{x})$ is to be found from Poisson's equation

$$
\frac{d^{2} \phi(\vec{x})}{d x^{2}}=-\frac{\rho(\vec{x})}{\varepsilon_{0}},
$$

and the electric field is given by

$$
E(\vec{x})=-\frac{d \phi(\vec{x})}{d x}
$$

In present simulation, it solves equations (1)-(6) along the collisionless trajectories for given boundary conditions.

The $1 d 3 v$ model of magnetized plasma sheath is shown schematically in Fig. 1. The simulation region considered is bounded by two parallel planes situated at $x=0$ and $x=L$ and the plasma consists of only electrons and singly charged ions. It has specified $x=L$ as the "sheath entrance" which separates the non-neutral, collision less sheath region $(x<L)$ from the quasineutral collisional presheath region $(x>L)$.

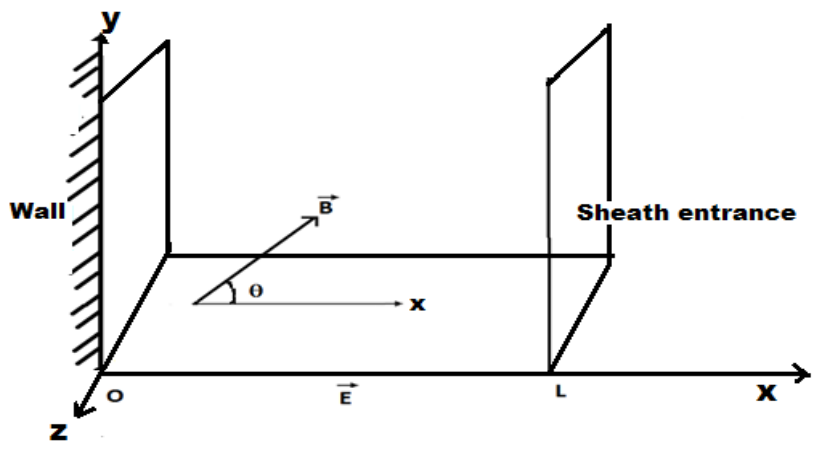

Fig. 1: Schematic diagram of the plasma sheath model
Similarly, an absorbing wall is specified by $x=0$. It is assumed that the angle made by the oblique magnetic field with the $x$-axis, which is the direction of the electric field, to be $\theta$. The magnetic field lies in the $x-y$ plane.

It is supposed that the plasma particles (electrons and ions) enter the simulation region from the sheath entrance with cut off Maxwellian velocity distribution functions, the wall doesn't emit any particles and that both boundaries are perfectly absorbing. Accordingly, the electron velocity distribution function is given by

$f^{e}(x, v)=A^{e} \exp \left[-\left(\frac{v_{x}^{2}+v_{y}^{2}+v_{z}^{2}}{v_{t f}^{e^{2}}}\right)+\frac{e \phi(\vec{x})}{k T_{e}}\right] \Theta\left[v_{c L}^{e}(x)-v_{x}\right]$

where $v_{c}^{e}(x)=\sqrt{\frac{2 e\left[\phi(x)-\phi_{0}\right]}{m^{e}}}$ is the electron cut off velocity at $x, k$ is the Boltzmann constant and $\Theta(\mathrm{x})$ is the Heaviside function i.e.,

$\Theta(x)=\begin{array}{ll}1 & \text { if } x \geq 0 \\ 0 & \text { otherwise }\end{array}$

The ion velocity distribution function at $x=L$ is given by,

$f^{i}(L, v)=A^{i} \exp \left[-\left(\frac{\left(v_{x}-v_{m L}^{i}\right)^{2}+v_{y}^{2}+v_{z}^{2}}{v_{t f}^{i^{2}}}\right)\right] \Theta\left(v_{c L}^{i}-v_{x}\right)$

where, $v_{t f}^{s}=\sqrt{\frac{2 k T^{s}}{m^{s}}}$ is the species-s (ion and electron) thermal velocity, $v_{m l}^{i}$ is the ion "Maxwellian-maximum" velocity at $x=L$ and $v_{c L}^{i}\left(v_{c L}^{i}<0\right)$ is the ion cut off velocity at $x=L$. In the core plasma the particle distribution would obviously be Maxwellian, however, in case of sheath formation the ions are accelerated towards the wall so that they become shifted Maxwellian as given by equation (9). In addition, for the Bohm criterion to be satisfied by the ions they must have attained certain minimum velocity $\left(v_{c L}^{i}\right)$ at the sheath entrance. As the electrons are retracted and reflected by the negative potential wall their distribution gets cut-off at the sheath entrance as given by equation (7).

Once all the starting parameters at the sheath entrance are known we solve the ion kinetic equations, for different discretized ion injection velocities up to the wall. This gives the ion velocities and their corresponding distribution function in the entire sheath region which on integration yields ion density distribution. For the velocity distribution given by equation (9), we can evaluate equation (3) for electron density at $x=L$ as

$n_{L}^{e}(x)=A^{e} \int_{=\infty}^{+\infty} d v_{x} \int_{-\infty}^{+\infty} d v_{y} \int_{-\infty}^{+\infty} d v_{z}\left[-\left(\frac{v_{x}^{2}+v_{y}^{2}+v_{z}^{2}}{v_{t f}^{e^{2}}}\right)\right] \Theta\left(v_{c L}^{e}-v_{x}\right)$ 
Now, from the velocity distribution function (9) and (3), we get ion density as

$n_{L}^{i}=A^{i} \int_{-\infty}^{+\infty} d v_{x} \int_{-\infty}^{+\infty} d v_{y} \int_{-\infty}^{+\infty} d v_{z}\left[-\left(\frac{\left(v_{x}-v_{m L}^{i}\right)^{2}+v_{y}^{2}+v_{z}^{2}}{v_{t f}^{i^{2}}}\right)\right] \Theta\left(v_{c L}^{i}-v_{x}\right)^{(11)}$

The average velocity for ions can be calculated by using

$u_{L}^{i}=\frac{1}{n_{L}^{i}} \int_{-\infty-\infty) \infty}^{v_{c}^{i}+\infty+\infty} \int_{-\infty}\left(v_{x} \hat{i}+v_{y} \hat{j}+v_{z} \hat{k}\right) \times A^{i} \exp \left[-\left(\frac{\left(v_{x}-v_{m L}^{i}\right)^{2}+v_{y}^{2}+v_{z}^{2}}{v_{t f}^{i^{2}}}\right)\right] d v_{x} d v_{y} d v_{z}$

and the average velocity for electrons is

$u_{L}^{e}=\frac{1}{n_{L}^{e}} \int_{-\infty-\infty-\infty}^{v_{c}^{e}+\infty+\infty} \int_{-\infty}\left(v_{x} \hat{i}+v_{y} \hat{j}+v_{z} \hat{k}\right) \times A^{e} \exp \left[-\left(\frac{v_{x}^{2}+v_{y}^{2}+v_{z}^{2}}{v_{t f}^{e^{2}}}\right)\right] d v_{x} d v_{y} d v_{z}$

The electron density profile, which is evaluated analytically, is given in terms of potential as

$$
n^{e}(\phi)=n_{L}^{e} \exp \left[\frac{e \phi(x)}{k T_{f}^{e}}\right]\left[\frac{1+e r f \sqrt{\frac{e\left(\phi(x)-\phi_{0}\right)}{k T_{f}^{e}}}}{1+e r f \sqrt{\frac{-e \phi_{0}}{k T_{f}^{e}}}}\right]
$$

\section{RESULTS}

The variation of various components of ion velocity with respect to time have been calculated numerically for magnetic field $2 \mathrm{mT}$ at different obliqueness of the field $\left(30^{\circ}, 60^{\circ}\right.$ and $\left.75^{\circ}\right)$ and the results are shown in Figs 2 to 4 . Figure 2 shows that at obliqueness $30^{\circ}$, the $x$-, $y$ - and $z$ component of velocity sinosodially varied about the mean value of $-106.9,6639$ and $11620 \mathrm{~ms}^{-1}$, respectively, with same frequency of oscillation $100 \mathrm{~Hz}$.

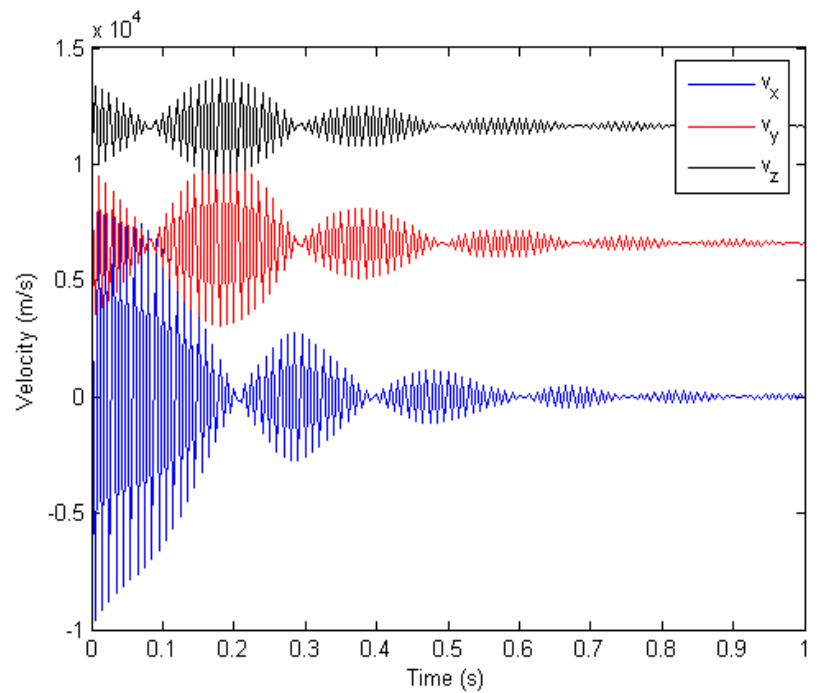

Fig. 2. Variation of velocity with time at magnetic field 2 $\mathrm{mT}$ and angle $30^{\circ}$
In addition, these components showed damping nature of oscillation with damping factor of $4.3,4.5$ and $4.2 \mathrm{~s}^{-1}$, respectively. Like wise at the angle of $30^{\circ}$, the $x$-, $y$ - and $z$ component of velocity varied with maximum amplitude of 9900.9, 3501 and $2070 \mathrm{~ms}^{-1}$, respectively. The figure shows that, for $x$-, $y$ - and $z$-component of velocity formed 5.5, 5.2 and 5.04 beats in $x$-, $y$ - and $z$-component of velocity in 1 second, so that their beat frequency was equal to 5.5, 5.2 and $5.04 \mathrm{~Hz}$. The first zero amplitude was obtained at about 0.2 second in $x$-component of velocity, whereas in $y$ - and $z$-component of velocity the first zero amplitude was obtained at around 0.1 second.

Figure 3 shows that at angle $60^{\circ}$, the $x$-, $y$ - and $z$ component of velocity sinosodially varied about the mean value of $-41.07,11590$ and $6683 \mathrm{~ms}^{-1}$, respectively, with constant frequency of oscillation $100 \mathrm{~Hz}$. In addition, like previous these components also showed damping nature of oscillation with damping factor $4.4,4.7$ and $4.2 \mathrm{~s}^{-1}$, respectively. Similarly, at the same angle, the $x$-, $y$ - and $z$ component of velocity varied with maximum amplitude 9835.07, 2630 and $4747 \mathrm{~ms}^{-1}$, respectively. Like wise this figure shows that for $x$-, $y$ - and $z$-component of velocity, there formed 5.3, 5.6 and 5.7 beats in 1 second so that their beat frequency was equal to $5.3,5.6$ and $5.7 \mathrm{~Hz}$. The first zero amplitude was obtained at around 0.15 second in $x$-component of velocity whereas in $y$ - and $z$-component of velocity the first zero amplitude was obtained at around 0.255 second.

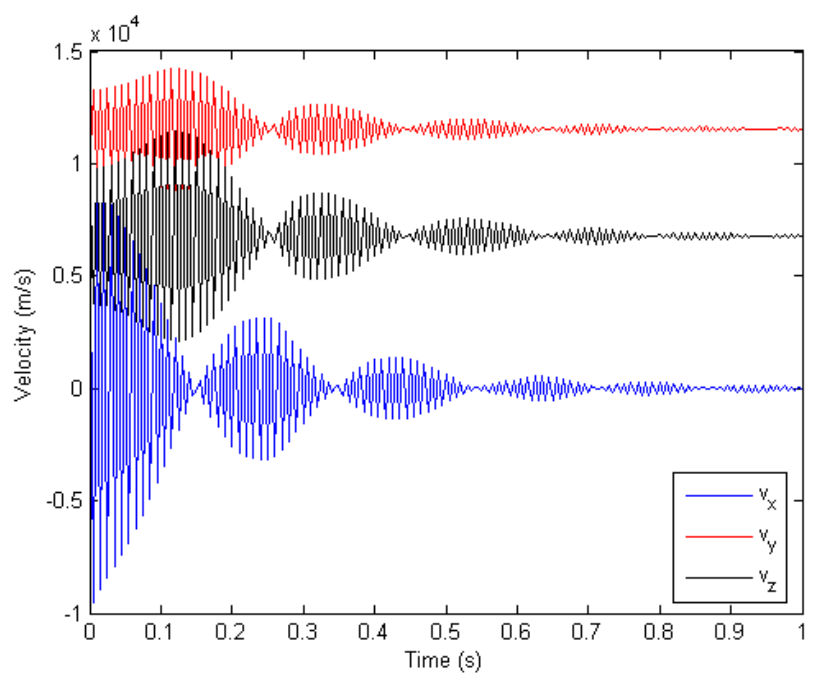

Fig. 3. Variation of velocity with time at magnetic field 2 $\mathrm{mT}$ and angle $60^{\circ}$

Figure 4 shows that the $x$-, $y$ - and $z$-component of velocity sinosodially varied about the mean value of 43.26, 11540 and $3296 \mathrm{~ms}^{-1}$, respectively, with frequency of oscillation $100 \mathrm{~Hz}$ at obliqueness $75^{\circ}$. In addition, these components showed damping nature of oscillation with damping factor of $4.5,4.2$ and $5.5 \mathrm{~s}^{-1}$, respectively. The $x$-, $y$ - and $z$ component of velocity varied with maximum amplitude 
$9750.74,1850$ and $6498 \mathrm{~ms}^{-1}$, respectively, at the same value of obliqueness.

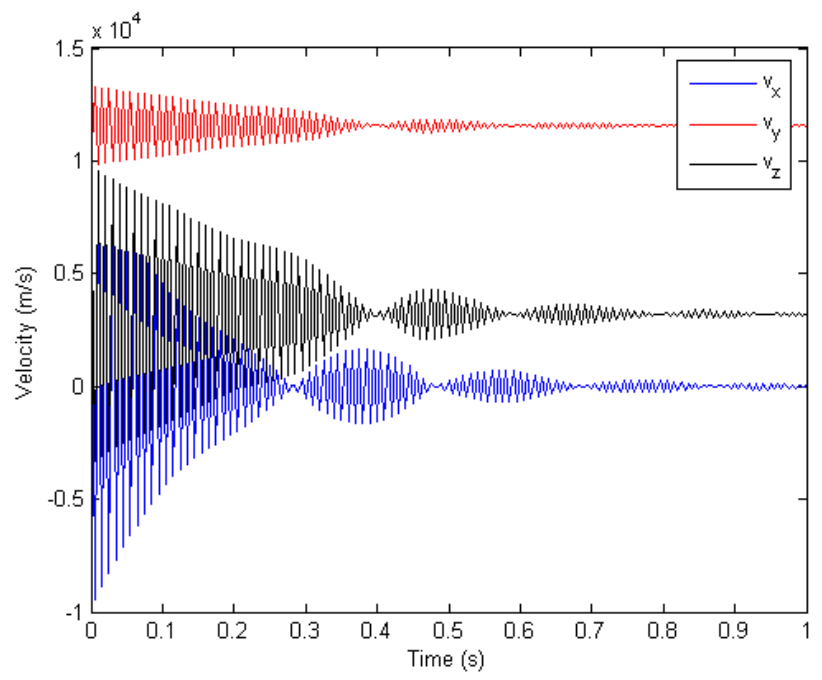

Fig. 4. Variation of velocity with time at magnetic field 2 $\mathrm{mT}$ and angle $75^{\circ}$
Similarly, Fig. 4 shows that at an angle $75^{\circ}$, for $x$-, $y$ - and $z$-component of velocity there formed 5.2, 5.6 and 5.6 beats in 1 second, respectively, so that their beat frequency was equal to $5.2,5.6$ and $5.6 \mathrm{~Hz}$, respectively. Also in $x$-component of velocity, the first zero amplitude was obtained at around 0.28 second whereas in $y$ - and $z$ component of velocity the first zero amplitude was obtained at around 0.4 second. Further in $x$-, $y$ - and $z$ component of velocity shoulder was obtained at around 0.1 second.

\section{DISCUSSION}

Table 1 shows the summary of observed data for mean value, maximum amplitude, damping constant, frequency of oscillation and beat frequency. The mean value of $x$ component of velocity at obliqueness of $30^{\circ}, 60^{\circ}$ and $75^{\circ}$ was small and negative compared to other components. The negative sign was due to the geometry considered where the wall is at origin and the ions enter the sheath region from $x=L$ with negative velocity. Mean value $\left(11620 \mathrm{~ms}^{-1}\right)$ was maximum of the $z$-component at $30^{\circ}$ with respect to all other components at different angles.

Table 1. The observed data of mean value, amplitude, damping constant, frequency of oscillation and beat frequency (all values are in SI units) at magnetic field 2 mT

\begin{tabular}{|c|c|c|c|c|c|c|c|c|c|c|c|c|c|c|c|}
\hline \multirow[t]{2}{*}{ Theta } & \multicolumn{3}{|c|}{ Mean Value } & \multicolumn{3}{|c|}{ Amplitude } & \multicolumn{3}{|c|}{$\begin{array}{l}\text { Damping } \\
\text { constant }\end{array}$} & \multicolumn{3}{|c|}{$\begin{array}{c}\text { Frequency of } \\
\text { oscillation }\end{array}$} & \multicolumn{3}{|c|}{ Beat frequency } \\
\hline & $\mathrm{v}_{\mathrm{xm}}$ & $\mathrm{v}_{\mathrm{ym}}$ & $\mathrm{v}_{\mathrm{zm}}$ & $\mathrm{v}_{\mathrm{xa}}$ & $\mathrm{v}_{\mathrm{ya}}$ & $\mathrm{V}_{\mathrm{za}}$ & $\mathrm{v}_{\mathrm{x}}$ & $\mathrm{v}_{\mathrm{y}}$ & $\mathrm{v}_{\mathrm{z}}$ & $\mathrm{v}_{\mathrm{x}}$ & $\mathrm{v}_{\mathrm{y}}$ & $\mathrm{v}_{\mathrm{z}}$ & $\mathrm{v}_{\mathrm{x}}$ & $\mathrm{v}_{\mathrm{y}}$ & $\mathrm{v}_{\mathrm{z}}$ \\
\hline $30^{\circ}$ & -106.9 & 6639 & 11620 & 9900.9 & 3501 & 2070 & 4.3 & 4.5 & 4.2 & 100 & 100 & 100 & 5.5 & 5.2 & 5.0 \\
\hline $60^{\circ}$ & -41.1 & 11590 & 6683 & 9835.1 & 2630 & 4747 & 4.4 & 4.7 & 4.2 & 100 & 100 & 100 & 5.3 & 5.6 & 5.7 \\
\hline $75^{\circ}$ & -43.3 & 11540 & 3296 & 9750.7 & 1850 & 6498 & 4.5 & 4.2 & 5.5 & 100 & 100 & 100 & 5.2 & 5.6 & 5.6 \\
\hline
\end{tabular}

Also at angle $75^{\circ}$ amplitude $\left(1850 \mathrm{~ms}^{-1}\right)$ was minimum of the $y$-component whereas at angle $30^{\circ}$ amplitude $(9900.9$ $\mathrm{ms}^{-1}$ ) was maximum of the $x$-component. At angle $60^{\circ}$ damping constant was maximum i.e. $4.7 \mathrm{~s}^{-1}$ of the $y$ component. The beat frequency along the $z$-component was maximum $(5.7 \mathrm{~Hz})$ for the angle $60^{\circ}$. As we were considering a uniform magnetic field the frequency of oscillation was same for all cases and presented here for the purpose of comparing with the beat frequency.

\section{CONCLUSION}

The beat nature shown by ion velocity in a uniform magnetized plasma sheath has been reported for the first time and has been analyzed for different obliqueness of the magnetic field. As the ions enter the sheath region they are guided by the magnetic field and hence start gyrating. As they move closer to the wall the electric field increases and the dominance of magnetic field ceases which causes the oscillation to have beat like nature. Thus the beat results primarily due to the combined effect of electric and magnetic fields. In present work we have studied the time evolution of ion velocity and the beat frequency for different obliqueness of the external uniform magnetic field. As the obliqueness of the field changes the separation of the mean values, beat frequency as well as the maximum amplitude of all the three component of the velocity changes. The study is useful in understanding the exact particle behavior when they travel in the sheath region and can be important in material processing, plasma etching and for confinement of plasma in fusion devices.

\section{ACKNOWLEDGEMENT}

One of the authors (BRA) would like to acknowledge the University Grants Commission-Nepal, Sanothimi, Nepal for Ph.D. Fellowship support.

\section{REFERENCES}

Bohm, D. 1949. The characteristics of electrical discharges in magnetic fields, Mc-Graw Hill, pp. 77.

Chalise, R. and Khanal, R. 2012. A kinetic trajectory simulation model for magnetized plasma sheath. 
Plasma Physisics and Controlled Fusion 54: 095006 (5pp).

Chalise, R. and Khanal, R. 2015. Self-consistent one dimension in space and three dimension in velocity kinetic trajectory simulation model of magnetized plasma-wall transition. Physics of Plasmas 22: $1135051-5$.

Chauhan, S., Ranjan, M., Bandyopadhyay, M. and Mukherjee, S. 2016. Droplet shaped anode double layer and electron sheath formation in magnetically constricted anode. Physics of Plasmas 23: 013502 19.

Chodura, R. 1982. Plasma wall transition in an oblique magnetic field. Physics of Fluids 25: 1628-1633.

Chodura, R. 1986. Physics of plasma-wall transition in controlled fusion. In: D. E. Post and R. Behrisch (eds), Plenum Publishing Corporation, pp. 99.

Gyergyek, T. and Kovacic, J. 2015. Fluid model of the sheath in front of a floating electrode immersed in a magnetized plasma with oblique magnetic field: Some comments on ion source terms and ion temperature effects. Physics of Plasmas 22: 0435021-0435024.

Hatami, M.M., Niknam, A.R., Shokri, B. and Ghomi, H. 2008. Magnetized plasma sheath with two species of positive ions. Physics of Plasmas 15: 053058 1-5.

Huang, C.W., Chen. Y.C. and Nishimura, Y. 2015. Particle-in-cell simulation of plasma sheath dynamics with kinetic ions. IEEE Transactions on Plasma Science 43: 675-682.

Khanal, R. 2003. A kinetic trajectory simulation (KTS) model for bounded plasma. Ph. D. Thesis, Innsbruck University, Austria.

Khoramabadi, M., Ghomi, H. and Shukla, P.K. 2011. Numerical investigation of the ion temperature effects on magnetized DC plasma sheath. Journal of Applied Physics 109: 073307 1-8.

Liberman, M.A., Lichtenberg, A.J., Kawamura, E. and Chabert, P. 2016. Linear electromagnetic excitation of an asymmetric low pressure capacitive discharge with unequal sheath widths. Physics of Plasmas 23(1): 013501-7.

Moulik, R. and Goswami, K.S. 2015. Potential around a dust grain in collisional plasma. Physics of Plasmas 22: 043701-6.

Riemann, K.U. 1991. The Bohm criterion and sheath formation. Journal of Physics D: Applied Physics 24: 493-518.

Riemann, K.U. 2000. Theory of the plasma-sheath transition The Bohm criterion and sheath formation. Journal of Technical Physics 41: 89-121.

Zou, X., Qiu, M., Liu, H., Zhang, L., Liu, J. and Gong, Y. 2009. The ion density distribution in a magnetized plasma sheath. Vacuum 83: 205-208. 